\title{
Population-Level Membrane Diversity Triggers Growth and Division of Protocells
}

\author{
Ö. Duhan Toparlak, Anna Wang, and Sheref S. Mansy*
}

Cite This: JACS Au 2021, 1, 560-568

Read Online

ABSTRACT: To date, multiple mechanisms have been described for the growth and division of model protocells, all of which exploit the lipid dynamics of fatty acids. In some examples, the more heterogeneous aggregate consisting of fatty acid and diacyl phospholipid or fatty acid and peptide grows at the expense of the more homogeneous aggregate containing a restricted set of lipids with similar dynamics. Imbalances between surface area and volume during growth can generate filamentous vesicles, which are typically divided by shear forces. Here, we describe another pathway for growth and division that depends simply on differences in the compositions of fatty acid membranes without additional components. Growth is driven by the thermodynamically favorable mixing of lipids between two populations, i.e., the system as a whole proceeds toward equilibrium. Division is the result of growth-induced curvature. Importantly, growth and division do not require a specific composition of lipids. For example, vesicles made from one type of lipid, e.g., short-chain fatty acids, grow and divide when fed with vesicles consisting of another type of lipid, e.g., long-chain fatty acids, and vice versa. After equilibration, additional rounds of growth and division could potentially proceed by the

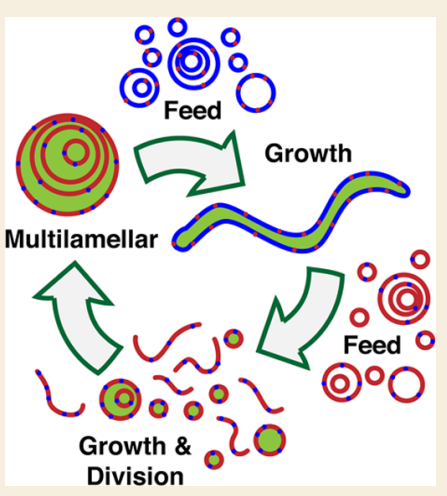
introduction of compositionally distinct aggregates. Since prebiotic synthesis likely gave rise to mixtures of lipids, the data are consistent with the presence of growing and dividing protocells on the prebiotic Earth.

KEYWORDS: Prebiotic chemistry, origins of life, fatty acid vesicles, protocell growth and division, artificial cells

\section{INTRODUCTION}

The formation of protocells, which is generally thought to involve the compartmentalization of nucleic acids, may have guided prebiotic chemistry toward systems capable of Darwinian evolution. ${ }^{1,2}$ If true, simple forms of growth and division likely existed to facilitate adaptation to changing environmental conditions and thus the persistence of the protocell. ${ }^{3,4}$ In the absence of complex metabolism and division machinery, early protocells would have been wholly reliant on chemical and physical forces for vital processes., ${ }^{5,6}$ For protocells built with fatty acids and their derivatives, ${ }^{6,7}$ several examples of growth and division have been reported. Alternative vesicle- ${ }^{8,9}$ and non-vesicle-based ${ }^{10-13}$ replication systems have also been described.

For protocells to grow, there must be a disequilibrium in the system. For instance, early examples of protocell growth relied on the addition of fatty acid esters, ${ }^{14}$ fatty anhydrides, ${ }^{15-18}$ or micelles. ${ }^{19,20}$ Here, growth either depended on the equilibration of fatty acid monomers (following the hydrolysis of fatty acid esters or anhydrides) with vesicles or the equilibration of excess micelles with vesicles. Both mechanisms benefit from the kinetic accessibility of the incorporation of monomers into existing vesicles, as opposed to the de novo production of vesicles. ${ }^{21-24}$ Alternatively, protocells can exploit disequilibria in osmotic pressures or lipid compositions. For example, osmotically swollen vesicles absorb lipids from isotonic vesicles to relieve membrane tension and hence gradients in chemical potential. ${ }^{25}$ Vesicles with lower membrane disorder can also grow at the expense of vesicles with higher membrane disorder. ${ }^{26,27}$ For these systems, only one component of the membrane could reach equilibrium after mixing, since the activation energy for the equilibration of the component with a lower desorption rate, either a diacyl phospholipid ${ }^{26}$ or hydrophobic peptide, ${ }^{27}$ was prohibitive. In general, for the systems explored thus far, the kinetics strongly favored the net flux of fatty acids toward one aggregate type.

Following growth, the division step of a growth-division cycle requires some form of mechanical force or bilayer instability. Gentle shear forces exerted on filamentous multilamellar vesicles ${ }^{20}$ or the extrusion of vesicles through pores $^{19}$ give rise to the formation of daughter vesicles. Vesicles can also divide through Rayleigh instabilities brought about by the generation of reactive oxygen species. ${ }^{28}$ Most investigations of the division of protocells start with a homogeneous

Received: November 16, 2020

Published: March 18, 2021 
compositions of lipids, although disequilibria can arise in some cases as a result of chemical reactivity. ${ }^{28}$

Prebiotic synthesis likely produced complex mixtures of lipids. ${ }^{29-31}$ Therefore, it is important to understand the impact of such heterogeneity. ${ }^{31-34}$ To do so, we sought to determine if the subtle kinetic differences between fatty acids of different chain lengths could, under some circumstances, impact population dynamics in such a way as to lead to the growth of vesicles. Here, we show that two distinct populations of model protocells, consisting of different fatty acids, can grow and divide, bringing us closer to understanding how a complete life cycle could have emerged. Protocells made with short-chain lipids drive the growth of protocells consisting of long-chain lipids, and long-chain-rich protocells induce the division of short-chain protocells (Scheme 1). The data, when coupled with the aforementioned work, suggest that growth and division were not rare phenomena on the prebiotic Earth. If protocells were composed of dynamic, nonkinetically trapped lipids, then growth and division could have been triggered when two populations of protocells with different membrane lipids mixed.

\section{RESULTS AND DISCUSSION}

\section{Growth and Division of Model Protocells}

To determine the impact of inhomogeneity between vesicle membranes, fatty acid vesicles consisting of lipids with different hydrophobic chain lengths were mixed and monitored by fluorescence microscopy. The two populations of vesicles were oleate (C18:1) multilamellar vesicles (MLVs) and myristoleate (C14:1) large unilamellar vesicles (LUVs). The MLVs were labeled with $0.15 \mathrm{~mol} \% \mathrm{~N}$-(lissamine rhodamine B sulfonyl)1,2-dihexadecanoyl-sn-glycero-3-phosphoethanolamine (LRDHPE), as previously reported. ${ }^{28}$ The MLVs were then extruded to $8 \mu \mathrm{m}$ and purified from vesicles that were less than $5 \mu \mathrm{m}$ in diameter with centrifugal filters. Unlabeled LUVs were extruded to $100 \mathrm{~nm}$. Fluorescence microscopy revealed that when these two populations of vesicles were mixed, the C18:1

Scheme 1. Protocellular Growth and Division
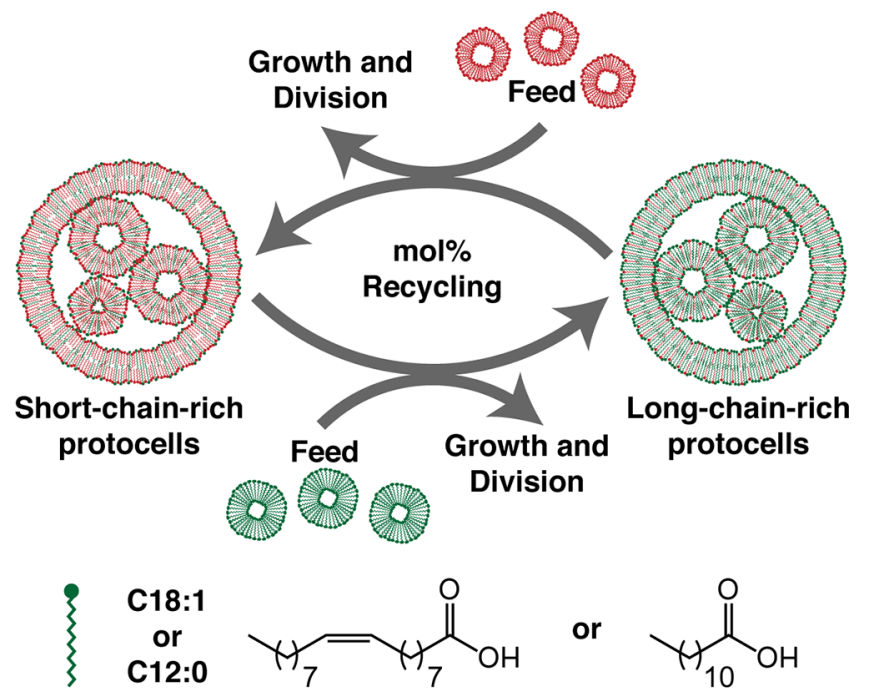

or<smiles>CCC(=O)O</smiles>

$\left\{\begin{array}{c}\text { C14:1 } \\ \text { or } \\ \text { C8:0 }\end{array}\right.$<smiles>CC(C)C=CC=CC(=O)O</smiles>

or<smiles>CCC(=O)O</smiles>

MLVs grew protrusions within $10 \mathrm{~min}$ and showed more extensive morphological changes after $60 \mathrm{~min}$ (Figures 1a and S1). No growth or morphological changes were visible for C18:1 MLVs when mixed with C18:1 LUVs (Figures 1b and S1), consistent with the light scattering studies of Cheng and Luisi. $^{35}$

To confirm that the MLVs grew through the incorporation of lipids from the LUVs, the surface area of the MLVs was monitored by Förster resonance energy transfer (FRET). The MLVs contained $0.3 \mathrm{~mol} \%$ of a FRET pair of fluorescently labeled lipids LR-DHPE and 1,2-dipalmitoyl-sn-glycero-3phosphoethanolamine- $N$-(7-nitro-2-1,3-benzoxadiazol-4-yl) (NBD-PE). ${ }^{36}$ The time-based change in surface area was extrapolated from known dye concentrations of MLVs (Figure S2a,b). C18:1 MLVs increased in surface area upon the addition of 6 equiv of C14:1 LUVs. This result was consistent with what was observed by fluorescence microscopy. More specifically, the surface area of the C18:1 MLVs increased 2fold within 2 min of mixing (Figure 1c) with no further changes observed after longer incubation. The data suggested that MLVs could grow when mixed with LUVs of a different lipid composition. This is different from past reports. Previously, the growth of pure fatty acid vesicles relied on

a
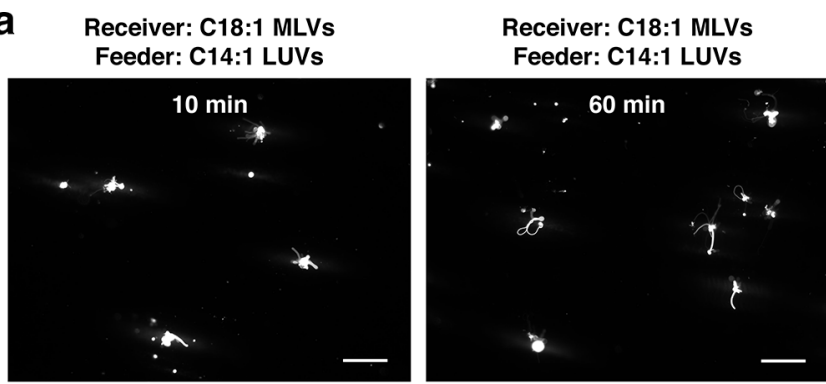

b

Receiver: C18:1 MLVs Feeder: C18:1 LUVs

Receiver: C18:1 MLVs

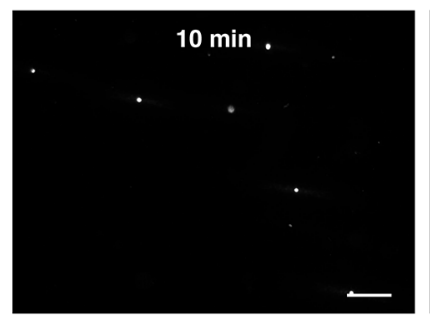

Feeder: C18:1 LUVs

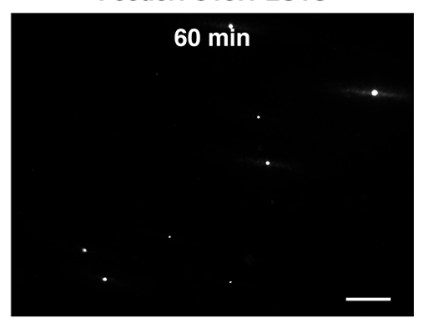

C

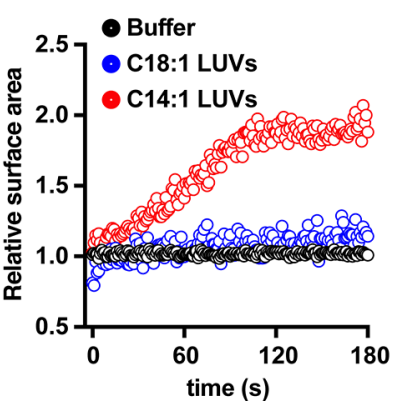

Figure 1. Heterogeneity in fatty acid composition induces growth. (a) Growth of C18:1 MLVs fed with 50 equiv of C14:1 LUVs after 10 and $60 \mathrm{~min}$. (b) C18:1 MLVs did not grow when fed with 50 equiv of C18:1 LUVs. (c) Surface area changes determined by FRET. C18:1 MLVs fed with 6 equiv of C14:1 LUVs showed an $\sim 2$-fold increase in surface area. Images in (a) and (b) are from different fields but with identical exposure times. Scale bars indicate $100 \mu \mathrm{m}$. 
feeding with micelles ${ }^{20,25,37}$ or fatty acid anhydrides ${ }^{17,18}$ of the same lipid composition (following hydrolysis of the anhydrides) as the vesicles, where the increased thermodynamic stability of vesicles over micelles and free monomers was responsible for growth under alkaline conditions. Instead, the growth of MLVs mixed with LUVs at concentrations far above the CVC was consistent with growth proceeding via a different mechanism.

Since C18:1 MLVs could grow by incorporating lipids from C14:1 LUVs, we wondered if the opposite was true as well, i.e., whether C14:1 MLVs could grow by feeding with C18:1 LUVs. To test this possibility, C14:1 MLVs were prepared with a fluorescently labeled lipid as described above for the C18:1 MLVs. Fluorescence microscopy did not immediately show obvious filamentous growth upon the addition of 5 equiv of C18:1 LUVs. However, many new smaller vesicles began to appear within the vicinity of the C14:1 MLVs (Figure 2a). The new smaller vesicles must have been produced from parent C14:1 MLVs, because only the C14:1 MLVs, not the C18:1 LUVs, were fluorescently labeled.

Such presumptive budding-off events were dependent on the concentration of the added C18:1 LUVs. Upon feeding, an immediate surge in the number of vesicles was detected by fluorescence microscopy (Figure S3 and Movies S1 and S2). Prolonged incubation led to a further increase in the number of daughter vesicles (Figure S3). Feeding with 1 equiv of C18:1 LUVs gave a decreased number of daughter vesicles with respect to feeding with 2.5 or 5 equiv of LUVs (Figure 2c) while also showing the presence of protrusions over $30 \mathrm{~min}$ (Figure S3b). No new vesicles were detected when C14:1 MLVs were fed with C14:1 LUVs (Figures $2 b$ and S4). The changes in the number of vesicles were further quantified by nanoparticle tracking analysis (NTA). The addition of 5 equiv of C18:1 LUVs to C14:1 MLVs gave an 5-fold increase in the number of vesicles within $10 \mathrm{~min}$ (Table S1). In addition to corroborating the microscopy data, the NTA data were able to detect the smaller vesicles not resolved by fluorescence microscopy.

To support the conclusion that division was induced by the uptake of the lipids, i.e., vesicle growth, the net surface area changes of C14:1 MLVs were monitored by FRET. Upon the addition of C18:1 LUVs, the surface area of C14:1 MLVs increased by $\sim 3$-fold within the first 2 min (Figure $2 \mathrm{~d}$ ). Unlike the growth of C18:1 MLVs (Figure 1c), the growth of C14:1 MLVs was biphasic (Figures 2d, S2d, and S3b). The surface area of feeder C18:1 LUVs decreased upon mixing with C14:1 MLVs, consistent with growth and division mediated by the uptake of C18:1 monomers (Figure S2d). The observed biphasic behavior suggested either the presence of multiple growth pathways ${ }^{37,38}$ or the growth of both the starting set of vesicles and the daughter vesicles (Figures S3b and S4b and Movies S1 and S2). Note that the total surface area would have increased regardless of whether the uptake of lipid led to division or not.

Taken together, MLVs made from either short-chain or long-chain lipids could grow when fed with LUVs of different lipid composition. However, growth and division required a significant difference in the characteristics of the lipid. For example, growth and division were not observed upon the mixing of vesicles of different but more similar lipid content. That is, fluorescence microscopy failed to reveal growth and division when C16:1 (palmitoleate) vesicles where mixed with C18:1 or C14:1 vesicles (Figure S5).
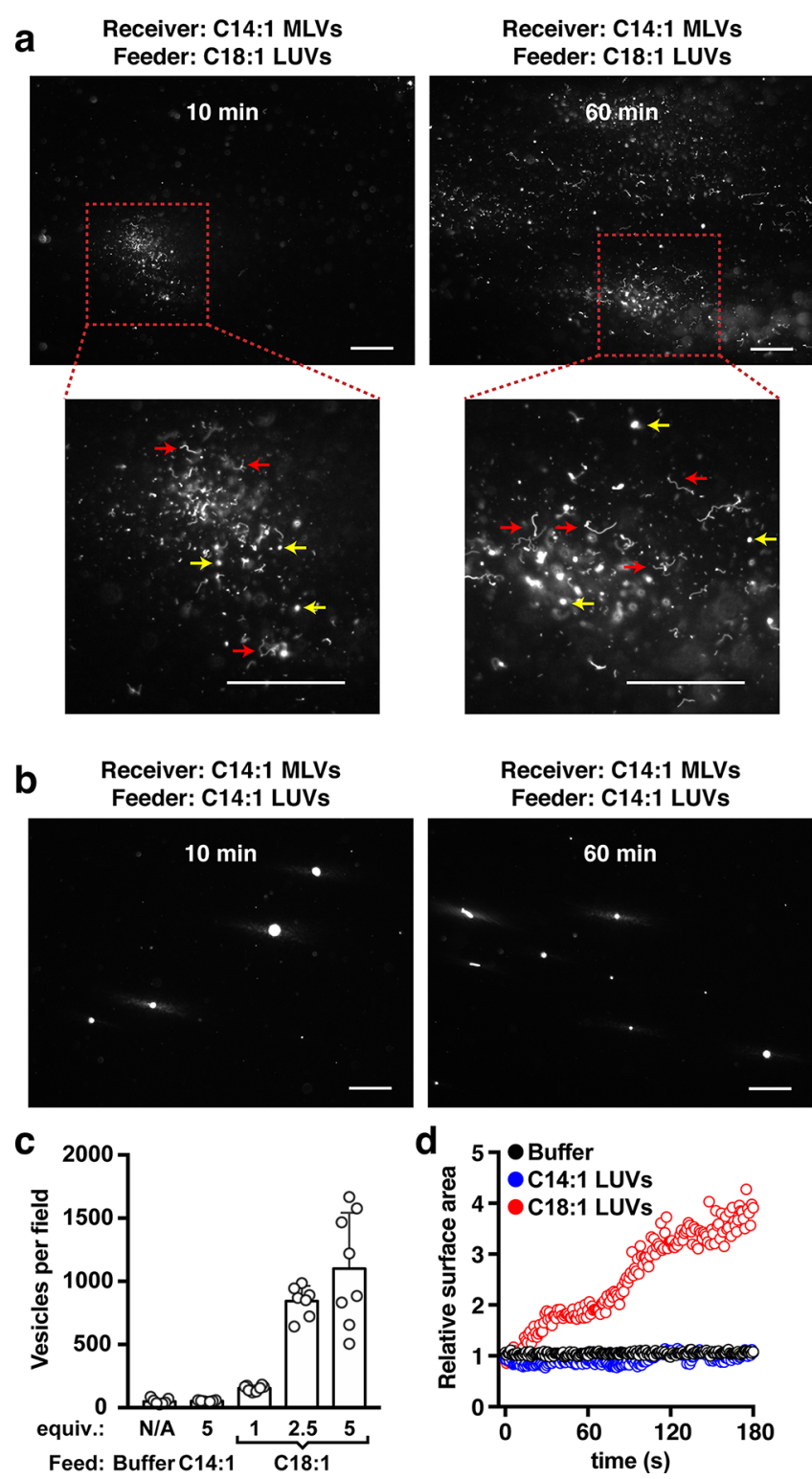

Figure 2. Heterogeneity in fatty acid composition induces growth and division. (a) C14:1 MLVs produced daughter vesicles when fed with 5 equiv of C18:1 LUVs. (b) C14:1 MLVs fed with 5 equiv of C14:1 LUVs did not show signs of growth or division. (c) Quantification of vesicle abundance. (d) Surface area changes determined by FRET. C14:1 MLVs fed with 2.5 equiv of C18:1 LUVs showed an $\sim 4$-fold increase in surface area within the first $3 \mathrm{~min}$. Images in (a) and (b) are from different fields but with identical exposure times. Red arrows indicate filamentous growth, and yellow arrows indicate freshly divided spherical vesicles. Scale bars indicate $100 \mu \mathrm{m}$. Flares are due to a long exposure time.

\section{Toward the Growth and Division of Prebiotically Plausible} Protocells

To determine if the observed growth and division of model protocells depended on the presence of LUVs, we next mixed fluorescently labeled, crude MLVs with MLVs extruded to 8 $\mu \mathrm{m}$ in the absence of LUVs. Here, fluorescently labeled, crude MLVs were mixed with MLVs extruded to $8 \mu \mathrm{m}$. Upon mixing, the C18:1 MLVs grew and divided when fed with 50 or 100 equiv of C14:1 crude MLVs (Figure 3). Subsequently, the resulting C14:1-rich mixture was subjected to another round of feeding by the addition of 4 equiv of C18:1 crude MLVs. 


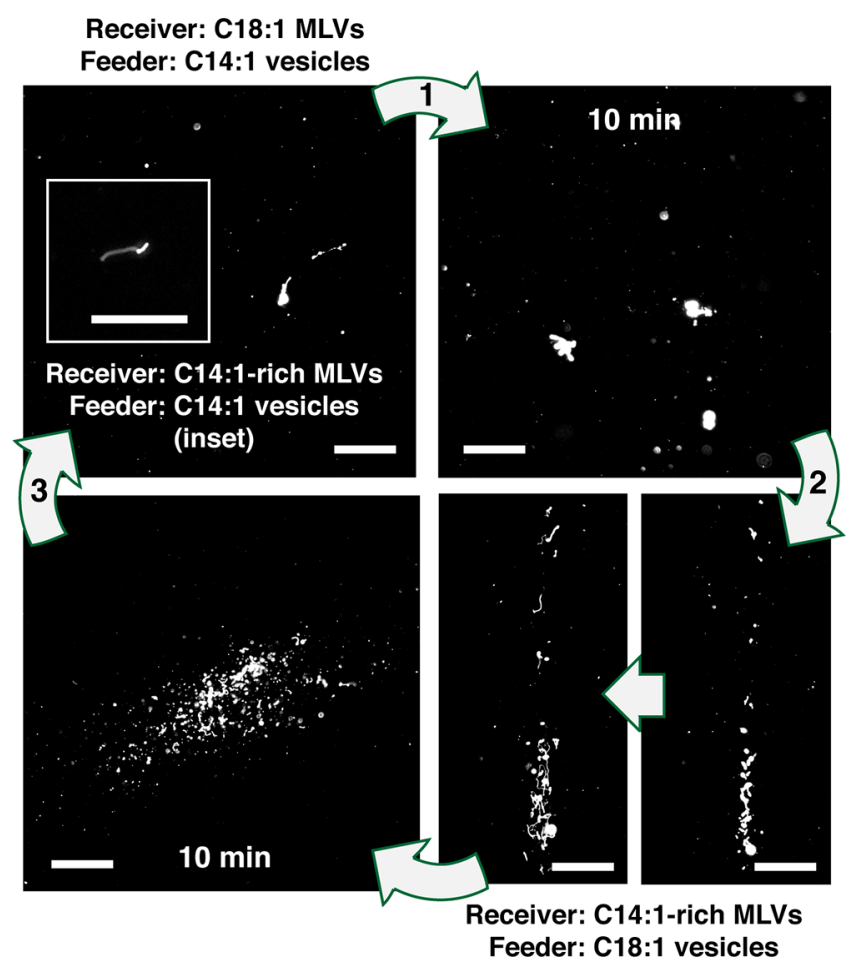

Figure 3. Growth and division of protocells with mixed compositions of bilayers. Scale bars indicate $100 \mu \mathrm{m}$.

Again, the vesicles grew and divided. Finally, C18:1 MLVs were added to the output from the second round of growth and division. Fluorescence microscopy indicated that the MLVs grew and divided (Figure 3). Therefore, iterative rounds of growth and division were possible with C18:1-rich and C14:1-rich vesicles.

Prebiotic chemistry likely produced mixtures of lipids of less than 14 carbons. ${ }^{29}$ To test if shorter, more prebiotically plausible lipids could give rise to the same behavior, laurate (C12:0) and caprylate (C8:0) vesicles were investigated. As expected, pure C12:0 MLVs (ca. 40 or $20 \mathrm{mM}$ ) grew when fed with 9 or 19 equiv of pure C8:0 vesicles (ca. 360 or $380 \mathrm{mM}$ ) (Figure 4a). To determine whether iterative growth and division was possible, the vesicles produced from mixing C12:0 MLVs with C8:0 LUVs (C8:0-rich vesicles) were further subjected to another round of growth and division by the addition of C12:0-rich crude vesicles. More specifically, the 19:1 caprylate/laurate MLVs (short-chain-rich MLVs) produced from the first round of growth and division were fed with 1 equiv of 1:19 caprylate/laurate LUVs (long-chain-rich) or 4 equiv of laurate vesicles. A nearly 2 -fold increase in the number of vesicles was detected after this second round of growth and division, as judged by fluorescence microscopy and vesicle counting (Figures $4 \mathrm{~b}, \mathrm{c}$ and S6). The data were consistent with that of the C18:1 and C14:1 mixtures (Figures $1-3)$, as the long-chain MLVs exhibited filamentous growth and the short-chain MLVs showed rapid division. The increase in the number of vesicles was of similar magnitude to the $\sim 3$ fold increase observed when C14:1 MLVs were fed with 1 equiv of C18:1 LUVs (Figure 2c). Overall, the observed behavior was dependent on a disparity in lipid composition between different vesicles and was not due to the presence of specific lipids. a
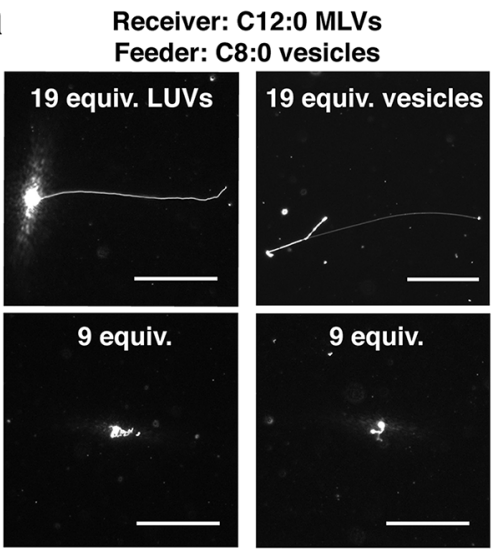

b Receiver: C12:0/C8:0 MLVs Feeder: C12:0 vesicles

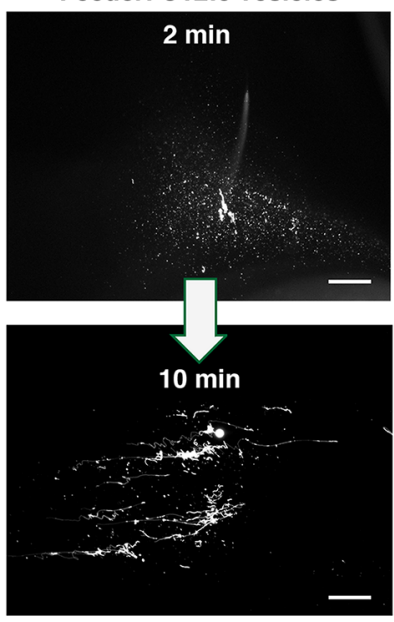

Receiver: C12:0/C8:0 MLVs Feeder: no feed

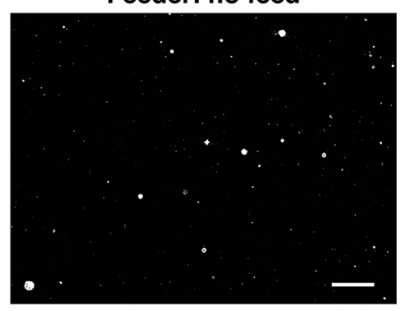

C

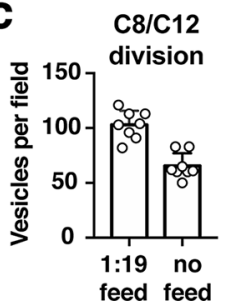

Figure 4. Growth and division with prebiotically plausible fatty acids. (a) Growth of C12:0 MLVs fed with C8:0 vesicles. Upper images were with 19 equiv of C12:0 vesicles, while lower images were with 9 equiv of C12:0 vesicles. (b) Growth and division of C8:0-rich (19:1 caprylate/laurate) MLVs fed with 1 equiv of C12:0-rich (1:19 caprylate/laurate) vesicles. (c) Quantification of vesicle abundance. Scale bars indicate $100 \mu \mathrm{m}$. Images were taken with identical exposure times. Imaging flares are due to a long exposure time.

\section{Retention of Entrapped Material}

Growing and dividing protocells would likely have aided the emergence of functional nucleic acids if the protocells were capable of retaining material. To probe the likelihood of such a scenario, crude mixtures of MLVs and LUVs were prepared in the presence of either calcein or fluorescently labeled DNA. After purification by size-exclusion chromatography, fresh LUVs were added to the purified mixture of MLVs and LUVs, and retention of encapsulant was quantified by an additional round of size-exclusion chromatography (Figure S7a,b). The starting population of C18:1 MLVs and LUVs retained 90\% of the encapsulated calcein after feeding with 50 equiv of C14:1 LUVs (Figure 5a). The same reaction with calcein loaded C18:1 LUVs in place of a mixture of C18:1 MLVs and LUVs gave similar results, $\sim 80 \%$ (Figure $5 \mathrm{~b}$ ). Mixtures of C14:1 MLVs and LUVs were less capable of retaining encapsulant when fed with C18:1 LUVs. The addition of 2.5 equiv of C18:1 LUVs led to an $\sim 50 \%$ loss of encapsulated DNA from a population of C14:1 MLVs and LUVs (Figure 5a). Retention was the same if the C14:1 MLVs were substituted with C14:1 LUVs. Receiver C14:1 vesicles lost 50\% 
a

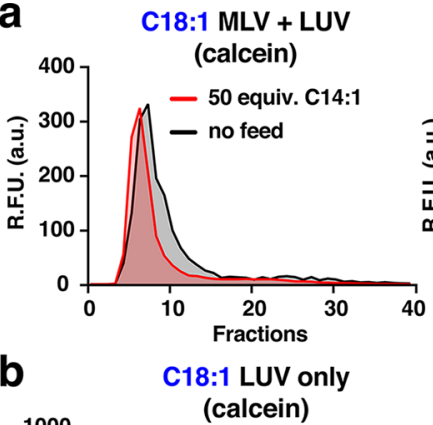

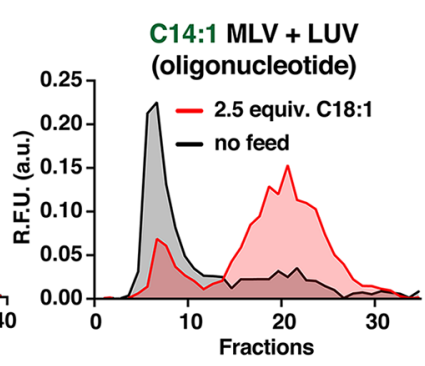

C14:1 LUV only (oligonucleotide)

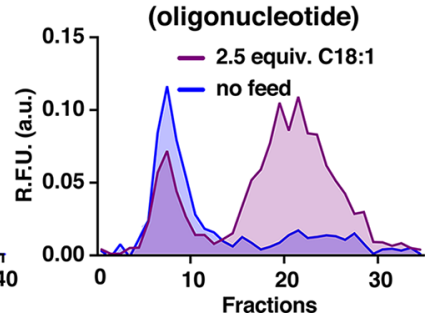

Figure 5. Retention of encapsulant during growth and division. (a) Size exclusion chromatography (SEC) of mixtures of MLVs and LUVs after the addition of LUVs. The encapsulant was either calcein or fluorescently labeled DNA. (b) SEC of fluorophore-containing LUVs after addition of unlabeled LUV. SEC was run 10 min after the addition of LUVs.

of the encapsulant in all cases, e.g., $\sim 60-70 \%$ was retained without feeding and 10-20\% was retained after feeding with C18:1 LUVs (Figures 5b and S7c,d). The reasons for the large loss of entrapped material were unclear and may have reflected rupture of a subset of the population, formation of large, transient pores, ${ }^{39}$ or other remodeling processes during division.

\section{Fatty Acid Dynamics}

Past work showed that disparities in lipid composition between fatty-acid-based membranes could drive the growth of vesicles. ${ }^{26}$ In these systems, kinetically trapped phospholipids could not equilibrate between bilayers. ${ }^{35}$ For the systems described herein, all of the lipids could dynamically equilibrate between all of the aggregates present. ${ }^{40}$ There were no kinetically trapped species, and a flux of lipids between membranes was always present. Nevertheless, kinetic differences must have existed, as the energetic cost of desorption and interleaflet flip-flop are impacted by the surface area of the hydrophobic chain. To confirm that differences in desorption rates were present, LUVs made from C14:1, C16:1, and C18:1 were mixed with 1-palmitoyl-2-oleoyl-sn-glycero-3-phosphocholine (POPC) vesicles containing a $\mathrm{pH}$-sensitive fluorophore (HPTS). In this assay, the rate of decrease in $\mathrm{pH}$ reflected the rate-limiting step, which was the desorption of the lipids from the LUVs (Figure S8a). ${ }^{41}$ As expected, the desorption rate was greatest for C14:1 followed by $\mathrm{C} 16: 1$ and $\mathrm{C} 18: 1$ (Figure 6a). The data were corroborated with POPC vesicles that contained FRET-labeled lipids in place of encapsulated HPTS (Figures $6 \mathrm{~b}$ and S8b). Rates of lipid flip-flop ${ }^{42}$ followed the same trend (Figures $6 \mathrm{c}$ and S8c). Finally, the ability of longer fatty acids to provide a more ordered intramembrane environment was confirmed by measuring the anisotropy of 1,6-diphenyl-1,3,5-hexatriene (DPH), a fluorophore embedded within the membrane (Figures 6d and S8d). Greater order within membranes is associated with decreased monomer offrates of lipids. ${ }^{43,26}$
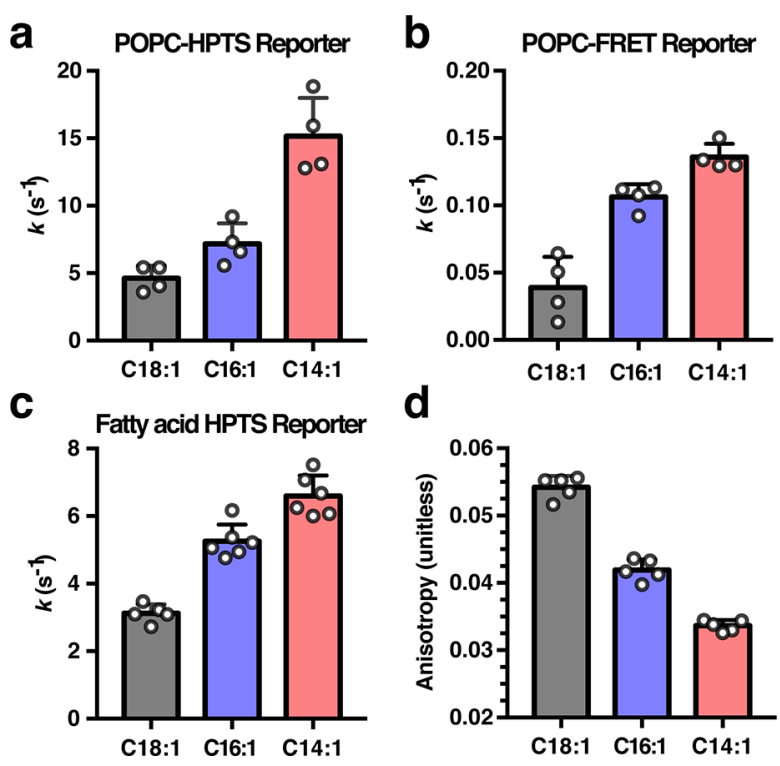

Figure 6. Lipid dynamics. Desorption rates determined by changes in $\mathrm{pH}$ (a) or FRET (b) of receiver POPC vesicles. (c) Rate of fatty acid flip-flop. (d) Fluorescence anisotropy of $\mathrm{DPH}^{34}$ within different membranes as an indicator of fluidity. All vesicles were LUVs. Data are mean with $\pm \mathrm{SD} ; n \geq 4$.

\section{Mechanism of Protocell Growth}

Although differences in lipid dynamics were present, these differences alone could not explain the observed behavior. Decreased rates of desorption of $\mathrm{C} 18: 1$ in comparison to C14:1 may have favored the growth of $\mathrm{C} 18: 1$ vesicles at the expense of $\mathrm{C} 14: 1$ vesicles. However, C14:1 vesicles were also able to grow at the expense of C18:1 vesicles. Instead, growth seemed to be primarily dependent on the greater displacement from equilibrium of the receiver MLVs upon mixing in comparison to the donor LUVs. In other words, donor LUVs were typically provided in excess, meaning that the concentration at equilibrium more closely resembled the starting concentration of lipids of the donor LUVs rather than the receiver MLVs. Therefore, the net direction of growth was not reliant on a specific composition of lipids as long as a disequilibrium existed. However, the different lipids must be sufficiently dissimilar in terms of their dynamics to mediate the behavior observed here.

Greater desorption kinetics suggest more facile equilibration with micellar aggregates, which exist in solutions containing fatty acid vesicles. As micelles are less thermodynamically favorable than vesicles above a critical concentration of lipid under specific solution conditions, the addition of micelles to vesicles results in growth. ${ }^{20,22,26,37,38}$ However, the system described herein did not show a dependence on a micellar phase, and LUVs and MLVs are equally stable. Large fatty acid vesicles of the same lipid composition but different diameters coexist without fusion and do not give rise to growth. ${ }^{35}$ For example, the concentration of lipid was well above the critical vesicle concentration (CVC) (Table S2), indicating that the fraction of micelles in solution was minor. Calculations ${ }^{44}$ indicated that the concentration of micelles increased upon the addition of C14:1 LUVs to C18:1 MLVs (CVC increased by more than 25-fold) and decreased upon the addition of C18:1 LUVs to C14:1 MLVs (decreased more than 25-fold).

Micelle-dependent growth was also not supported by kinetic measurements. Assuming that growth of acceptor membranes 
was first order with respect to feeder vesicles, we extracted the effective rate constants of growth from the FRET data in Figures 1 and 2 (Figure S9a,b). The growth rate of C14:1 MLVs upon feeding with C18:1 LUVs $(k=0.014 / \mathrm{s})$ was one order of magnitude greater than for C18:1 MLVs upon the addition of C14:1 LUVs $(k=0.0014 / \mathrm{s})$. Both growth rates were much slower than the rates observed by micelle addition. $^{37}$ Taken together, these observations further bolstered the hypothesis that membrane growth in our system occurred via pathways other than simple micelle-driven monomer adsorption.

We then sought to understand the morphological changes following growth. Others have observed that a rapid increase in surface area to volume ratio can lead to nonequilibrium structures consisting of lobes and protrusions. ${ }^{45,46}$ The resulting lobes and protrusions are intrinsically unstable, since these structures deviate from the thermodynamically favorable spheroid form, ultimately leading to division. ${ }^{47,48}$ When the buffer (HEPES), which crosses the membrane slowly, was replaced with the much more permeable ammonium acetate, ${ }^{20}$ we still observed shape changes, albeit to a lesser extent (Figure S10). These results suggest that the rate of membrane growth in our system could still outpace volume growth when the lipid tails differed by four carbon units, highlighting how small, subtle differences in molecular structure can lead to large changes at the population level.

\section{Mechanism of Protocell Division}

We then used a simple kinetic model to determine how our system could build up enough curvature to aid division. We found that the addition of 5 equiv of C18:1 LUVs to C14:1 MLVs generated curvature equivalent to that of $70 \mathrm{~nm}$ diameter vesicles (Figure S9c), thus favoring very thin structures and consistent with our observations. Conversely, the addition of 4 equiv of C16:1 LUVs to C14:1 MLVs generated curvature equivalent to $100 \mathrm{~nm}$ diameter vesicles, which may be enough to induce a slight degree of growth but not division (Figures S9c and S5). The addition of 5 equiv of C14:1 feeder LUVs to C18:1 MLVs generated curvature equivalent to that of $1.6 \mu \mathrm{m}$ diameter vesicles (Figure S9d). Similarly, the addition of 5 equiv of C16:1 feeder LUVs to C18:1 MLVs generated curvature equivalent to that of $1 \mu \mathrm{m}$ diameter vesicles (Figure S9d). These curvatures are likely inadequate to favor the formation of thin, filamentous structures. The addition of 50 equiv of C14:1 LUVs to C18:1 MLVs, however, generated curvature equivalent to that of $160 \mathrm{~nm}$ diameter vesicles (Figure S9e), which would likely favor filament formation; again, consistent with our observations. Following the formation of high-curvature structures, it is possible that C18:1 and C14:1 MLVs divided via different mechanisms. Unlike the mechanisms described for diacyl phospholipids, ${ }^{46,49-52}$ the fatty acid lipids used here possessed similar curvature. Therefore, division likely proceeded through the dynamic accumulation of lipid in the outer leaflet that outpaced relaxation processes. ${ }^{53}$ The resulting positive curvature would then lead to division via rupture or membrane remodeling. However, a better theoretical understanding of bilayer kinetics is necessary to delineate the exact mechanism of division when vesicles of different lipid compositions are mixed and would entail more extensive biophysical characterization.

\section{CONCLUSIONS}

Prebiotic conditions must have produced protocells that were heterogeneous in composition. Rather than inhibiting the emergence of functional systems, mixtures of lipids likely aided the formation of protocellular structures. Mixtures of lipids increase the stability and modulate the permeability of protocellular membranes. ${ }^{33,44,54,55}$ Furthermore, as we show here, mixing different populations of protocells can lead to multiple, iterative rounds of growth and division under specific conditions. However, for sustained cycles of growth and division to take place, mechanisms are needed to keep the system away from equilibrium and to maintain the composition of the protocell. Although physical mechanisms, e.g., the mixing of rivulets, ${ }^{56}$ could be envisaged to support the growth and division of the compartment, entrapped material such as nucleic acids would be continually diluted after each growth-division cycle unless supported by an internal metabolism. $^{30,5759}$ The leakiness of primitive membranes and disruptions to membrane integrity due to the division pathways would have placed an even greater selective pressure on the system for such protometabolic support. Additionally, heterogeneous protocellular populations of different size, lamellarity, and composition would have led to growth and division processes running in parallel.

Although the differences in lipid composition that were used to drive growth were based on differences in the hydrophobic chains, computational models suggest that similar effects are likely to arise from differences in the lipid headgroup. ${ }^{59}$ Modifications of the headgroup would be more chemically accessible $\mathrm{e}^{31,34,58-60}$ and thus would more easily tie into the chemical activity of the protocell. Since vesicles are capable of retaining entrapped nucleic acids during growth and division, such systems may have the potential to support Darwinian evolution. ${ }^{61,62}$ Perhaps more importantly, what is described herein is a new model for coupled growth and division that is in addition to previously discovered mechanisms. With so many possibilities, it seems reasonable to conclude that growing and dividing vesicles were likely present on the prebiotic Earth.

\section{EXPERIMENTAL SECTION}

For full experimental details, please see the Supporting Information. Preparation and Purification of Protocells

All vesicles were prepared by either the hydration of a thin film or the dispersion of a neat fatty acid oil. Organic solvents used to dissolve the lipids were either chloroform for phospholipids or 9:1 methanol/ chloroform for fluorescently labeled phospholipids. Briefly, desired amounts of lipids from stock solutions were pipetted into glass vials and evaporated under a gentle flow of $\mathrm{N}_{2}$. Then, mixtures were dried by house vacuum overnight $(<16 \mathrm{~h})$ to remove residual solvent. The next day, the vesicles were hydrated with buffers or buffers containing fluorophore, vortexed briefly multiple times $(4-5 \mathrm{~s})$, and then left to equilibrate with tumbling for $<24 \mathrm{~h}$. For C14:1, C16:1, and C18:1 vesicles, the buffer was $0.2 \mathrm{M} \mathrm{Na}^{+}$-HEPES, $\mathrm{pH} 8.0$, with the $\mathrm{pH}$ adjusted with $5 \mathrm{M} \mathrm{NaOH}$. After tumbling, vesicles were extruded with $100 \mathrm{~nm}$ or $8 \mu \mathrm{m}$ track-etched polycarbonate membranes using an Avanti miniextruder (at least 11 passes). The extruded vesicles were left undisturbed in the dark overnight $(16-24 \mathrm{~h})$. For C8:0 and C12:0 vesicles, the buffer was $1 \mathrm{M} \mathrm{Na}^{+}$-HEPES, $\mathrm{pH}$ 7.0, with the $\mathrm{pH}$ adjusted with $5 \mathrm{M} \mathrm{NaOH}$. The vesicles of $\mathrm{C} 8: 0$ and $\mathrm{C} 12: 0$ were constantly kept above $55^{\circ} \mathrm{C}$ with a conductive metal heat block, and these experiments were completed within $2 \mathrm{~h}$ after preparation of the vesicles. If needed, the vesicles were purified by size exclusion chromatography, with lipid concentrations kept above the CVC in the 
running buffer. Fractions were collected with a Gilson FC204B multichannel fraction collector, analyzed with a Tecan M200 (BioTek) or a Varioskan (ThermoFisher) plate reader. All purified vesicles were used within 24-72 h of the final extrusion.

\section{Growth and Division of Protocells}

Multilamellar vesicles were prepared as described above and were tagged with $0.15 \mathrm{~mol} \% \mathrm{~N}$-(lissamine rhodamine B sulfonyl)-1,2dihexadecanoyl-sn-glycero-3-phosphoethanolamine (LR-DHPE). Following tumbling and extrusion, the small vesicles with $<5 \mu \mathrm{m}$ size were removed by multiple cycles of brief centrifugation with spin column filters (Ultrafree-MC-Durapore $5 \mu \mathrm{m}$ with PVDF membrane, Millipore) while keeping the overall lipid concentration above the CVC with vesicles of the same composition. Feeder vesicles were also prepared as above and used after equilibration for $24 \mathrm{~h}$ except for the C8:0 and C12:0 lipids, which were used within $2 \mathrm{~h}$ of preparation. For iterative growth, the vesicles were mixed and equilibrated for $10 \mathrm{~min}$ and then imaged.

\section{FRET Assay for Vesicle Surface Area Change}

Pure and mixed membrane growth kinetics were measured taking previous reports as a guide. ${ }^{19,20,22,25-28}$ The surface area changes were monitored at $\lambda_{\mathrm{ex}}=430 \mathrm{~nm}$ and $\lambda_{\mathrm{em}}=580 \mathrm{~nm}$ with FRET donor/ acceptor pairs, 1,2-dipalmitoyl-sn-glycero-3-phosphoethanolamine- $N$ (7-nitro-2-1,3-benzoxadiazol-4-yl) (NBD-PE) and $\mathrm{N}$-(lissamine rhodamine B sulfonyl)-1,2-dihexadecanoyl-sn-glycero-3-phosphoethanolamine (LR-DHPE). The total mol \% of FRET-labeled lipids was between 0.2 to $0.4 \mathrm{~mol} \%$, and the relative surface area changes were extrapolated using a calibration curve for fluorescence readouts of known fluorophore concentrations. Triton X-100 (1\% (v/v, final)) was used to disrupt the vesicles to assess background levels.

\section{Determination of Interleaflet Flip-Flop Kinetics}

Single-chain amphiphile vesicles were prepared as above to encapsulate $5 \mathrm{mM}$ 8-hydroxypyrene-1,3,6-trisulfonic acid (HPTS) in $0.2 \mathrm{M} \mathrm{Na}^{+}$-HEPES, $\mathrm{pH} 8.0$, and mixed in a stopped-flow device with $1: 1(\mathrm{v} / \mathrm{v}) \quad 0.2 \mathrm{M} \mathrm{Na}^{+}$-HEPES, 7.0. Fluorescence was monitored continuously with $\lambda_{\mathrm{ex}}=454 \mathrm{~nm}$ and $\lambda_{\mathrm{em}}=515 \mathrm{~nm}$ for $10-200$ points/s.

\section{Determination of Desorption Rates}

The $\mathrm{pH}$-sensitive fluorophore HPTS was used to monitor the decay of the $\mathrm{pH}$ gradient across the membrane of reporter unilamellar POPC vesicles, as previously established. ${ }^{41,42}$ Fatty acid vesicles were prepared as above, and POPC vesicles encapsulated $5 \mathrm{mM}$ HPTS in $0.2 \mathrm{M} \mathrm{Na}^{+}$-HEPES, $\mathrm{pH}$ 8.0. The two vesicle populations were mixed in a stopped-flow device, and the fluorescence measurements were taken with $\lambda_{\mathrm{ex}}=454 \mathrm{~nm}$ and $\lambda_{\mathrm{em}}=515 \mathrm{~nm}$ and $100-200$ points $/ \mathrm{s}$. Alternatively, reporter unilamellar POPC vesicles contained $0.2 \mathrm{~mol} \%$ of FRET-pair lipids. The raw data was fit to a single-phase decay $F(t)$ $=F(\infty)+F(0) \exp (-t / \tau)$ to determine the experimental time constant $\tau=t_{\text {obs }}$. Then the $t_{1 / 2}$ was calculated as $\ln (2)\left(t_{\text {obs }}\right)$, and $k_{\text {off }}$ was calculated as $1 / t_{\text {obs. }}$.

\section{Modeling of Bilayer Curvature}

The model used considers the number of lipid molecules in the feeder vesicles $(F)$ and on the outer $(O)$ and inner $(I)$ leaflets of the receiver vesicles. This model captures the earlier time points of the experiment, when the system is far from equilibrium and the rate of membrane growth, $k_{a}$, can be assumed to be constant. At the earlier time points, excess lipid accumulating on the outer leaflet generates curvature stress and is transported from the outer to inner leaflets via flip-flop at rate $k_{\mathrm{f}}$.

$$
\begin{aligned}
& \mathrm{d} O / \mathrm{d} t=k_{\mathrm{a}} F-k_{\mathrm{f}}(O-I) \\
& \mathrm{d} I / \mathrm{d} t=k_{\mathrm{f}}(O-I) \\
& \mathrm{d} F / \mathrm{d} t=-k_{\mathrm{a}} F
\end{aligned}
$$

These equations are integrated numerically with a time step of $50 \mathrm{~ms}$. The rates of curvature relaxation $k_{\mathrm{f}}$ are taken from the literature $\left(k_{\mathrm{f}}=\right.$ $0.6 \mathrm{~s}^{-1}$ for C18:1, $k_{\mathrm{f}}=1.0 \mathrm{~s}^{-1}$ for C16:1, and $k_{\mathrm{f}}=1.5 \mathrm{~s}^{-1}$ for C14:1). ${ }^{53}$

\section{ASSOCIATED CONTENT}

\section{Supporting Information}

The Supporting Information is available free of charge at https://pubs.acs.org/doi/10.1021/jacsau.0c00079.

Figure S1. Growth of oleate multilamellar vesicles (C18:1 MLVs) fed with myristoleate large unilamellar vesicles (C14:1 LUVs). Figure S2. FRET-based vesicle surface area monitoring. Figure S3. Division of myristoleate multilamellar vesicles (C14:1 MLVs) fed with oleate large unilamellar vesicles (C18:1 LUVs). Figure S4. C14:1 MLV protocell growth and divisions with varying ratios of oleate $(\mathrm{C} 18: 1)$ and myristoleate (C14:1) vesicles. Figure S5. Growth and division studies with C16:1 vesicles. Figure S6. Additional images on divided vesicles of prebiotically plausible lipids. Figure S7. Experimental workflow and analysis of protocells encapsulating calcein, following growth and division. Figure S8. Demonstration of fatty acid behavior for characterization of fatty acid monomers within and between vesicles. Figure S9. Curve-fitting and modeling analyses for protocell growth and division. Figure S10. Protocell growth under various conditions. Table S1. Fluorescently labeled MLV concentration change during growth and division, fed with LUVs. Table S2. Theoretical calculations for final lipid (at equilibrium) and critical vesicle concentration (CVC) changes in protocells upon mixing (PDF)

Movie S1. Spontaneous division of C14:1 MLVs after feeding with 1 equiv of C18:1 LUVs (within first seconds) (AVI)

Movie S2. Spontaneous division of C14:1 MLVs after feeding with 1 equiv of C18:1 LUVs (after first seconds) (AVI)

\section{AUTHOR INFORMATION}

\section{Corresponding Author}

Sheref S. Mansy - Department of Cellular, Computational and Integrative Biology (D-CIBIO), University of Trento, 38123 Povo, TN, Italy; Department of Chemistry, University of Alberta, Edmonton, AB T6G 2G2, Canada; 10 orcid.org/ 0000-0003-2382-198X; Email: sheref.mansy@ualberta.ca

\section{Authors}

Ö. Duhan Toparlak - Department of Cellular, Computational and Integrative Biology (D-CIBIO), University of Trento, 38123 Povo, TN, Italy; 이이이.org/0000-0002-0859-1373

Anna Wang - School of Chemistry and Australian Centre for Astrobiology, UNSW Sydney, Sydney, NSW 2052, Australia; - orcid.org/0000-0002-2148-1996

Complete contact information is available at: https://pubs.acs.org/10.1021/jacsau.0c00079

\section{Author Contributions}

Ö.D.T. performed the wet-lab experiments. A.W. performed the in silico modeling. Ö.D.T. and S.S.M. conceived the project, designed the experiments, and wrote the manuscript together with contribution from A.W. S.S.M. supervised the study. All authors have given approval to the final version of the manuscript. 


\section{Notes}

The authors declare no competing financial interest.

\section{ACKNOWLEDGMENTS}

Funding from the Simons Foundation (290358FY18 and 290358FY19) is acknowledged. This project received funding from the European Union's Horizon 2020 research and innovation programme under grant agreement No 824060 . We thank J. W. Szostak and R. Krishnamurthy for valuable comments on the manuscript.

\section{REFERENCES}

(1) Szostak, J. W.; Bartel, D. P.; Luisi, P. L. Synthesizing Life. Nature 2001, 409, 387-390.

(2) Hanczyc, M. M.; Monnard, P.-A. Primordial membranes: more than simple container boundaries. Curr. Opin. Chem. Biol. 2017, 40, $78-86$.

(3) Mansy, S. S. Membrane Transport in Primitive Cells. CSH Perspect. Biol. 2010, 2, No. a0022188.

(4) Pearce, S.; Perez-Mercader. PISA: construction of self-organized and self-assembled functional vesicular structures. Polym. Chem. 2021, $12,29-49$.

(5) Blain, C. J.; Szostak, J. W. Progress Toward Synthetic Cells. Annu. Rev. Biochem. 2014, 83, 615-640.

(6) Gebicki, J. M.; Hicks, M. Ufasomes are Stable Particles surrounded by Unsaturated Fatty Acid Membranes. Nature 1973, 243, 232-234.

(7) Hargreaves, W. R.; Deamer, D. W. Liposomes from Ionic, SingleChain Amphiphiles. Biochemistry 1978, 17, 3759-3768.

(8) Takakura, K.; Toyota, T.; Sugawara, T. A Novel System of SelfReproducing Giant Vesicles. J. Am. Chem. Soc. 2003, 125, 8134-8140.

(9) Liu, L.; Zou, Y.; Bhattacharya, A.; Zhang, D.; Lang, S. Q.; Houk, K. N.; Devaraj, N. K. Enzyme-free synthesis of natural phospholipids in water. Nat. Chem. 2020, 12, 1029-1034.

(10) Bandela, A. K.; Wagner, N.; Sadihov, H.; Morales-Reina, S.; Chotera-Ouda, A.; Basu, K.; Cohen-Luria, R.; de la Escosura, A.; Ashkenasy, G. Primitive selection of the fittest emerging through functional synergy in nucleopeptide networks. Proc. Natl. Acad. Sci. U. S. A. 2021, 118 (9), No. e2015285118.

(11) Ottelé, J.; Hussain, A. S.; Mayer, C.; Otto, S. Chance emergence of catalytic activity and promiscuity in a self-replicator. Nat. Catal. 2020, 3, 547-553.

(12) Omosun, T. O.; Hsieh, M.-C.; Childers, W. S.; Das, D.; Mehta, A. K.; Anthony, N. R.; Pan, T.; Grover, M. A.; Berland, K. M.; Lynn, D. G. Catalytic diversity in self-propagating peptide assemblies. Nat. Chem. 2017, 9, 805-809.

(13) Saghatelian, A.; Yokobayashi, Y.; Soltani, K.; Ghadiri, M. R. A chiroselective peptide replicator. Nature 2001, 409, 797-801.

(14) Bachmann, P. A.; Luisi, P. L.; Lang, J. Autocatalytic selfreplicating micelles as models for prebiotic structures. Nature 1992, $357,57-59$.

(15) Zepik, H. H.; Blöchliger, E.; Luisi, P. L. A Chemical Model of Homeostasis. Angew. Chem., Int. Ed. 2001, 40 (1), 199-201.

(16) Berclaz, N.; Müller, M.; Walde, P.; Luisi, P. L. Growth and Transformation of Vesicles Studied by Ferritin Labeling and Cryotransmission Electron Microscopy. J. Phys. Chem. B 2001, 105 (5), 1056-1064.

(17) Walde, P.; Wick, R.; Fresta, M.; Mangone, A.; Luisi, P. L. Autopoietic Self-Reproduction of Fatty Acid Vesicles. J. Am. Chem. Soc. 1994, 116, 11649-11654.

(18) Wick, R.; Walde, P.; Luisi, P. L. Light microscopic investigations of the autocatalytic self-reproduction of giant vesicles. J. Am. Chem. Soc. 1995, 117, 1435-1436.

(19) Hanczyc, M. M.; Fujikawa, S. M.; Szostak, J. W. Experimental Models of Primitive Cellular Compartments: Encapsulation, Growth and Division. Science 2003, 302, 618-622.
(20) Zhu, T. F.; Szostak, J. W. Coupled Growth and Division of Model Protocell Membranes. J. Am. Chem. Soc. 2009, 131, 57055713.

(21) Budin, I.; Bruckner, R. J.; Szostak, J. W. Formation of Protocelllike Vesicles in a Thermal Diffusion Column. J. Am. Chem. Soc. 2009, 131, 9628-9629.

(22) Budin, I.; Debnath, A.; Szostak, J. W. Concentration-driven Growth of Model Protocell Membranes. J. Am. Chem. Soc. 2012, 134, 20812-20819.

(23) Hentrich, C.; Szostak, J. W. Controlled growth of filamentous fatty acid vesicles under flow. Langmuir 2014, 30, 14916-14925.

(24) Albertsen, A. N.; Maurer, S. E.; Nielsen, K. A.; Monnard, P.-A. Transmission of photo-catalytic function in a self-replicating chemical system: in situ amphiphile production over two protocell generations. Chem. Commun. 2014, 50, 8989-8992.

(25) Chen, I. A.; Roberts, R. W.; Szostak, J. W. The Emergence of Competition Between Model Protocells. Science 2004, 305, 14741476.

(26) Budin, I.; Szostak, J. W. Physical Effects Underlying the Transition from Primitive to Modern Cell Membranes. Proc. Natl. Acad. Sci. U. S. A. 2011, 108, 5249-5254.

(27) Adamala, K.; Szostak, J. W. Competition between model protocells driven by an encapsulated catalyst. Nat. Chem. 2013, 5, 495-501.

(28) Zhu, T. F.; Adamala, K.; Zhang, N.; Szostak, J. W. Photochemically Driven Redox Chemistry Induces Protocell Membrane Pearling and Division. Proc. Natl. Acad. Sci. U. S. A. 2012, 109, 9828-9832.

(29) McCollom, T. H.; Ritter, G.; Simoneit, B. R. T. Lipid Synthesis Under Hydrothermal Conditions by Fischer-Tropsch-Type Reactions. Origins Life Evol. Biospheres 1999, 29, 153-166.

(30) Bonfio, C.; Caumes, C.; Duffy, C. D.; Patel, B. H.; Percivalle, C.; Tsanakopoulou, M.; Sutherland, J. D. Length-Selective Synthesis of Acylglycerol-Phosphates through Energy-Dissipative Cycling. J. Am. Chem. Soc. 2019, 141, 3934-3939.

(31) Fiore, M.; Madanamoothoo, W.; Berlioz-Barbier, A.; Maniti, O.; Girard-Egrot, A.; Buchet, R.; Strazewski, P. Giant vesicles from rehydrated crude mixtures containing unexpected mixtures of amphiphiles formed under plausibly prebiotic conditions. Org. Biomol. Chem. 2017, 15, 4231.

(32) Jordan, S. F.; Rammu, H.; Zheludev, I. N.; Hartley, A. M.; Maréchal, A.; Lane, N. Promotion of Protocell Self-assembly from Mixed Amphiphiles at the Origin of Life. Nat. Ecol. Evol. 2019, 3, $1705-1714$.

(33) Cape, J. C.; Monnard, P.-A.; Boncella, J. M. Prebiotically relevant mixed fatty acid vesicles support anionic solute encapsulation and photochemically catalyzed trans-membrane charge transport. Chem. Sci. 2011, 2, 661-671.

(34) Fayolle, D.; Altamura, E.; D'Onofrio, A.; Madanamothoo, W.; Fenet, B.; Mavelli, F.; Buchet, R.; Stano, P.; Fiore, M.; Strazewski, P. Crude phosphorylation mixtures containing racemic lipid amphiphiles self-assemble to give stable primitive compartments. Sci. Rep. 2017, 7, 18106.

(35) Cheng, Z.; Luisi, P. L. Coexistence and Mutual Competition of Vesicles with Different Size Distributions. J. Phys. Chem. B 2003, 107 (39), 10940.

(36) Struck, D. K.; Hoekstra, D.; Pagano, R. E. Use of Resonance Energy Transfer to Monitor Membrane Fusion. Biochemistry 1981, 20, 4093-4099.

(37) Chen, I. A.; Szostak, J. W. A Kinetic Study of the Growth of Fatty Acid Vesicles. Biophys. J. 2004, 87, 988-998.

(38) Chen, I. A.; Szostak, J. W. Membrane Growth Can Generate a Transmembrane pH Gradient in Fatty Acid Vesicles. Proc. Natl. Acad. Sci. U. S. A. 2004, 101, 7965-7970.

(39) Karatekin, E.; Sandre, O.; Guitouni, H.; Borghi, N.; Puech, P.H.; Brochard-Wyart, F. Cascades of Transient Pores in Giant Vesicles: Line Tension and Transport. Biophys. J. 2003, 84 (3), 1734-1749.

(40) Luisi, P. L. Are Micelles and Vesicles Chemical Equilibrium Systems? J. Chem. Educ. 2001, 78, 380-384. 
(41) Zhang, F.; Kamp, F.; Hamilton, J. A. Dissociation of Long and Very Long Chain Fatty Acids from Phospholipid Bilayers. Biochemistry 1996, 35, 16055-16060.

(42) Kamp, F.; Hamilton, J. A. pH Gradients across Phospholipid Membranes Caused by Fast Flip-flop of Un-ionized Fatty Acids. Proc. Natl. Acad. Sci. U. S. A. 1992, 89, 11367-11370.

(43) Shinitzky, M.; Barenholz, Y. Fluidity Parameters of Lipid Regions Determined by Fluorescence Polarization. Biochim. Biophys. Acta, Rev. Biomembr. 1978, 515, 367-394.

(44) Budin, I.; Prywes, N.; Zhang, N.; Szostak, J. W. Chain-length Heterogeneity allows for the Assembly of Fatty Acid Vesicles in Dilute Solutions. Biophys. J. 2014, 107, 1582-1590.

(45) Mui, B. L.; Cullis, P. R.; Evans, E. A.; Madden, T. D. Osmotic Properties of Large Unilamellar Vesicles Prepared by Extrusion. Biophys. J. 1993, 64 (2), 443-453.

(46) Kindt, J. T.; Szostak, J. W.; Wang, A. Bulk self-assembly of giant, unilamellar vesicles. ACS Nano 2020, 14, 14627.

(47) Lipowsky, R. Coupling of Bending and Stretching Deformations in Vesicle Membranes. Adv. Colloid Interface Sci. 2014, 208, 1424.

(48) Ruiz-Herrero, T.; Fai, T. G.; Mahadevan, L. Dynamics of Growth and Form in Prebiotic Vesicles. Phys. Rev. Lett. 2019, 123, 038102 .

(49) Han, S. Molecular dynamics simulation of oleic acid/oleate bilayers: an atomistic model for a ufasome membrane. Chem. Phys. Lipids 2013, 175-176, 79-83.

(50) Chen, C.-M.; Higgs, P. G.; MacKintosh, F. C. Theory of Fission for Two-Component Lipid Vesicles. Phys. Rev. Lett. 1997, 79, 1579.

(51) Sakuma, Y.; Imai, M. Model System of Self-Reproducing Vesicles. Phys. Rev. Lett. 2011, 107, 198101.

(52) Urakami, N.; Jimbo, T.; Sakuma, Y.; Imai, M. Molecular mechanism of vesicle division induced by coupling between lipid geometry and membrane curvatures. Soft Matter 2018, 14, 30183027.

(53) Bruckner, R. J.; Mansy, S. S.; Ricardo, A.; Mahadevan, L.; Szostak, J. W. Flip-Flop Induced Relaxation of Bending Energy: Implications for Membrane Remodeling. Biophys. J. 2009, 97, 31133122.

(54) Mansy, S. S.; Schrum, J. P.; Krishnamurthy, M.; Tobé, S.; Treco, D.; Szostak, J. W. Template-Directed Synthesis of a Genetic Polymer inside of a Model Protocell. Nature 2008, 454, 122-125.

(55) Mansy, S. S.; Szostak, J. W. Thermostability of Model Protocell Membranes. Proc. Natl. Acad. Sci. U. S. A. 2008, 105, 13351-13355.

(56) Patel, B. H.; Percivalle, C.; Ritson, D. J.; Duffy, C. D.; Sutherland, J. D. Common origins of RNA, protein and lipid precursors in a cyanosulfidic protometabolism. Nat. Chem. 2015, 7, 301-307.

(57) Sutherland, J. D. Opinion: Studies on the Origin of Life - the End of the Beginning. Nat. Rev. Chem. 2017, 1, 0012.

(58) Bonfio, C.; Russell, D. A.; Green, N. J.; Mariani, A.; Sutherland, J. D. Activation chemistry drives the emergence of functionalised protocells. Chem. Sci. 2020, 11, 10688.

(59) Piedrafita, G.; Monnard, P.-A.; Mavelli, F.; Ruiz-Mirazo, K. Permeability-driven selection in a semi-empirical protocell model: the roots of prebiotic systems evolution. Sci. Rep. 2017, 7, 3141.

(60) Toparlak, Ö. D.; Karki, M.; Egas Ortuno, V.; Krishnamurthy, R.; Mansy, S. S. Cyclophospholipids Increase Protocellular Stability to Metal Ions. Small 2020, 16, 1903381.

(61) Engelhart, A. E.; Adamala, K. P.; Szostak, J. W. A Simple Physical Mechanism Enables Homeostasis in Primitive Cells. Nat. Chem. 2016, 8, 448-53.

(62) O’Flaherty, D. K.; Kamat, N. P.; Mirza, F. N.; Li, L.; Prywes, N.; Szostak, J. W. Copying of Mixed-Sequence RNA Templates inside Model Protocells. J. Am. Chem. Soc. 2018, 140, 5171-51. 